\title{
Advanced Development Techniques for Metal-Based EUV Resists
}

\author{
Jodi Hotalen, ${ }^{\mathrm{a}}$ Michael Murphy, ${ }^{\mathrm{a}}$ William Earley, ${ }^{\mathrm{a}}$ Michaela Vockenhuber, ${ }^{\mathrm{b}}$ Yasin Ekinci, \\ Daniel A. Freedman, ${ }^{c}$ and Robert L. Brainard ${ }^{\mathrm{a}}$ \\ a. SUNY Polytechnic Institute - CNSE, Albany, NY 12203 \\ b. Paul Scherrer Institut, Villigen, Switzerland \\ c. State University of New York at New Paltz, New Paltz, NY 12561
}

\begin{abstract}
Pure thin-films of unimolecular organometallic photoresists were lithographically evaluated using extreme ultraviolet light (EUV, $\lambda=13.5 \mathrm{~nm}$ ) and developed using solutions containing carboxylic acids. Optimization of development solutions used with a cobalt-oxalate EUV resist (NP1, 2) led to a switch in lithographic tone from negative to positive. Additional optimization led to an improvement in top loss (35 to 7\%) with development in cyclohexanone and 2-butanone, respectively. We saw a drastic improvement in photo-speed $\left(\mathrm{E}_{\max }=5 \mathrm{~mJ} / \mathrm{cm}^{2}\right)$ and contrast of the negative-tone imaging with development in certain acidic solutions. Additionally, carboxylic acid solutions provide excellent development conditions for resists that we, in the past, have been unable to successfully develop.
\end{abstract}

Keywords: extreme ultraviolet, organometallic, resist, positive-tone, photoresist, contrast, cobalt, oxalate.

\section{INTRODUCTION}

One of the challenges of traditional organic resists is the low absorbance of extreme ultraviolet photons (EUV, $13.5 \mathrm{~nm}$ ). As resist films are coated thinner to prevent pattern collapse, even fewer photons are absorbed in the resist film which results in worsened stochastics. ${ }^{1,2}$ As a result, metal-based photoresists show strong promise for future use in EUV lithography since transition and post-transition metals absorb far more EUV photons. ${ }^{3}$ Doug Keszler at the Oregon State University (OSU) and Chris Ober at Cornell University demonstrated that hafnium oxide (films or nanoparticles) could act as good EUV photoresists. ${ }^{4}$ Jason Stowers et al. published results with inorganic resists showing high resolution and good sensitivity in 2009. ${ }^{5}$ Chris Ober's research team has been focused on evaluating the lithographic performance of metal-oxide nanoparticles. ${ }^{6}$ In 2015, Inpria published on implementing their Yseries metal-based resist systems into fab operation processes for EUV lithography. ${ }^{7}$ Metal-based resists have much different photochemistry than organic resists and consequently the development process yields unique solubility differences for each system.

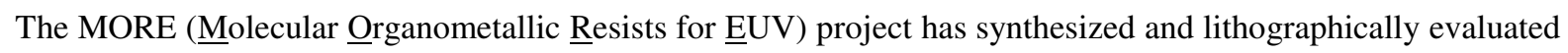
over 1000 potential resists with varying metal centers, around twelve different optically dense metals have been investigated. Of those twelve, our group has focused on seven platforms of metal-based resists since 2011 involving cobalt, ${ }^{8}$ tin, ${ }^{9}$ palladium, ${ }^{10}$ bismuth ${ }^{11}$ and antimony. ${ }^{12}$ We have proposed that one strategy for creating positive-tone organometallic resists is through the use of oxalate ligands. The first positive-tone resists of this type were oxalate complexes with platinum and palladium (1) centers. ${ }^{6}$ During exposure, the oxalate ligand $\left(\mathrm{C}_{2} \mathrm{O}_{4}{ }^{2-}\right)$ is converted to carbon dioxide $\left(\mathrm{CO}_{2}\right)$ which was confirmed by mass spectroscopy. ${ }^{13}$ When this very polar ligand is removed, the resulting photoproduct is less polar creating a solubility difference between the exposed and unexposed regions. Our first successful MORE resists is the compound benzyltriphenylphosphonium bipyridinebis(oxalate)cobaltate(III) (NP1, 2) which originally was developed with methylethylketone (MEK) providing negative-tone imaging. This compound also contains oxalate ligands and has similar photochemistry to the platinum and palladium oxalates in

Extreme Ultraviolet (EUV) Lithography VIII, edited by Eric M. Panning, Kenneth A. Goldberg, Proc. of SPIE Vol. 10143, 1014309 - (c) 2017 SPIE · CCC code: 0277-786X/17/\$18 · doi: 10.1117/12.2258126 
that the exposure eliminates the ligand as carbon dioxide. ${ }^{8}$ Prior to the work described here, we have seen only negative-tone imaging with NP1.

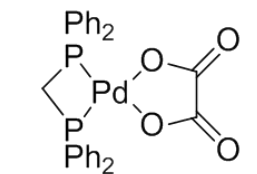

1

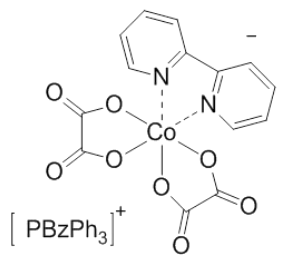

2
Positive Tone $\mathrm{E}_{\min }=\sim 85 \mathrm{~mJ} / \mathrm{cm}^{2}$

Negative Tone $E_{\max }=36 \mathrm{~mJ} / \mathrm{cm}^{2}$

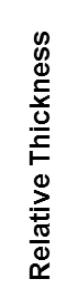
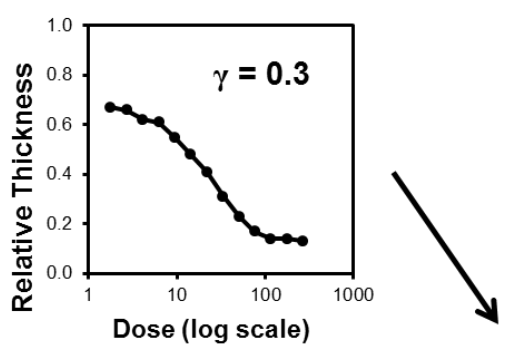<smiles>O=[Y10]1oc(=O)c(=O)o1</smiles>

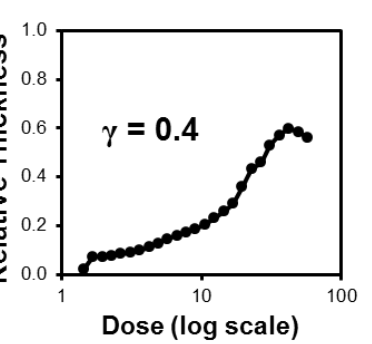

Figure 1. First positive-tone MORE results were with platinum- and palladium-oxalate complexes (1). The cobalt-oxalate complex (2), known as NP1, has a faster photo-speed, but produces negative-tone imaging when developed in pure organic solvents. We attempted to combine the best features of each platform into one resist.

The focus of the MORE project has been to synthesize new resists and understand their photochemistry and, until recently, we have spent very little time optimizing process conditions (e.g. development and post exposure bakes). Generally, our organometallic resists were developed in pure organic solvents or pure water, and until this work, the use of acidic or basic solutions was unexplored. We can use the development step to cause chemical reactions which enhance the solubility of the resist.

Here, we describe the use of carboxylic acid solutions as developers for metal-based resists. These developers can improve the performance of both positive- and negative-tone imaging. In this work, successful positive-tone results with the cobalt-oxalate complex were achieved using these specialized developers with better photosensitivity than previous positive-tone MORE resists. Additionally, some of these acidic developers improved the photoresist contrast, the rate of change in film thickness as a function of logarithmic dose, of the negative-tone imaging of the same cobalt-oxalate resist. Lastly, we found that solutions of carboxylic acids could provide excellent development conditions for certain resists (antimony-based) that were insoluble in pure organic solvents after exposure to vacuum. We compare the lithographic performance of our metal-based resists to an open source resist (OS2), which is a traditional chemically amplified EUV photoresist. ${ }^{14}$

\section{RESULTS AND DISCUSSION}

\subsection{Acidic Development: Positive-Tone Imaging of Cobalt Complexes}

From prior work, the proposed photomechanism assumes the liberation of the oxalate ligand in the form of carbon dioxide $\left(\mathrm{CO}_{2}\right)$ from the cobalt-oxalate resist, during which the cobalt center is reduced from $\mathrm{Co}^{\mathrm{III}}$ to $\mathrm{Co}^{\mathrm{II}} .8$ This mechanism is supported by mass spectrometry, infrared spectroscopy, cyclic voltammetry, and paramagnetic NMR data. ${ }^{8,13}$ Unoccupied coordination sites on the cobalt center can be filled by lone pairs of electrons on neighboring carbonyl oxygen atoms creating an insoluble coordination polymer, $\mathrm{Co}(\mathrm{II})(\mathrm{bpy})(\mathrm{ox})(\mathbf{4}){ }^{15}$ 


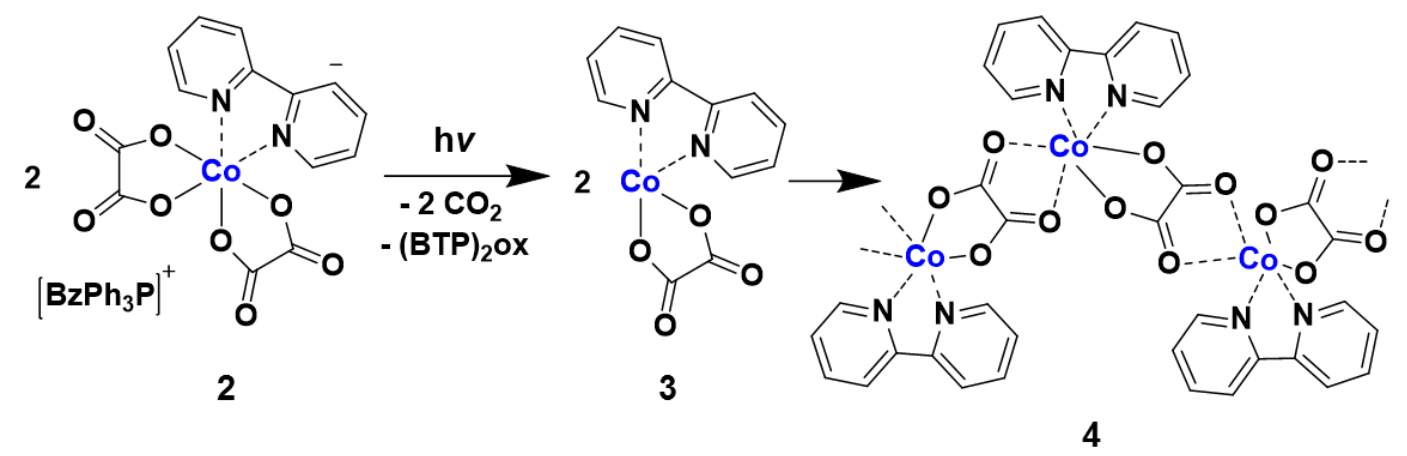

Figure 2. Proposed photomechanism of NP1 (2). The suspected photoproduct (3) is a reduced version which has open coordination sites that can be filled by lone pair electrons on nearby oxygen atoms creating an insoluble network (4) of $\mathrm{Co}(\mathrm{II})(\mathrm{bpy})(\mathrm{ox})$ in the exposed regions. ${ }^{15}$

Having some knowledge of the photomechanism and photoproducts of NP1, we suspected that we could convert this resist from negative- to positive-tone imaging. We propose that the oxalate bridging could be either prevented or broken to create a soluble photoproduct and convert NP1 to a positive-tone resist (Figure 3). In Pathway A, a ligand (such as an amine) is formulated into the resist film that, upon elimination of the oxalate ligand during the exposure, can fill the coordination sites before the lone pair electrons on the carbonyl oxygen. We found that adding amines (ligands) to the formulation produced poor coatings. Additionally, adding an amine to the resist formulation would reduce the optical density of the film. The concept in Pathway B is that once the insoluble coordination polymer is formed, by adding a ligand or reactive species to the developer we can break the oxalate bridging and increase solubility. This avenue proved more effective than Pathway A.

(A)

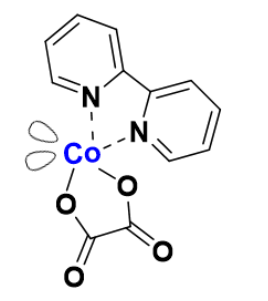

3

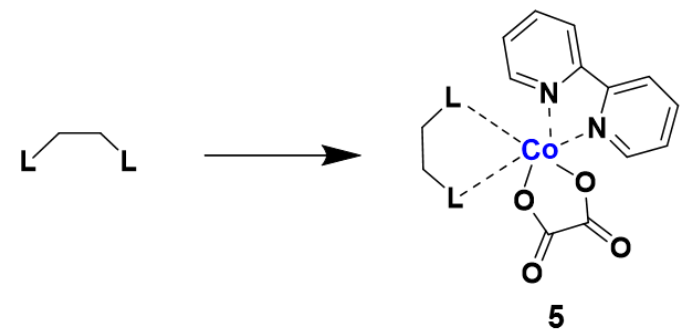

(B)

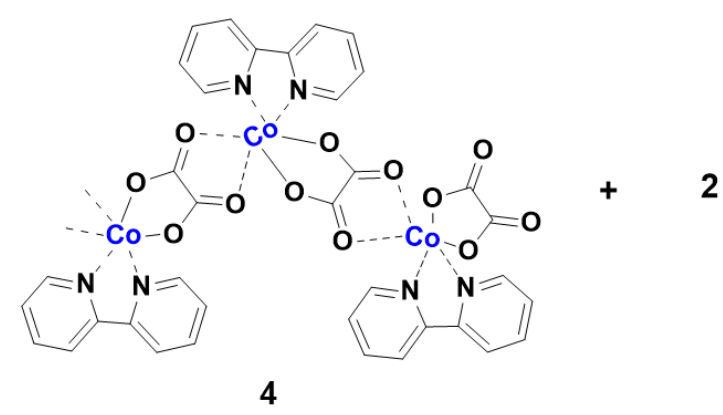

$2 \mathrm{~L}$

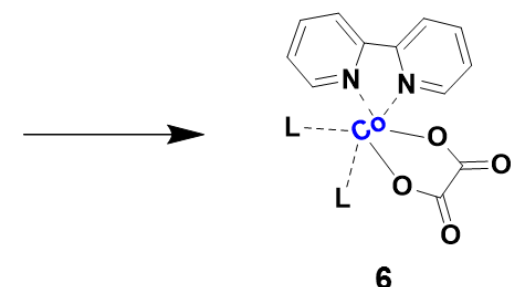

Figure 3. Two approaches to create a soluble compound(s) in the exposed regions to convert this cobalt-oxalate compound from a negative- to a positive-tone resist.

Modification of the developer resulted in successful positive-tone imaging of NP1 by adding a small amount of a carboxylic acid, specifically acetic acid (Figure 4). After the initial discovery, a diverse selection of organic acids was studied (Figure 5). 
Concept:

The amine could fill the open coordination sites on the cobalt, preventing the insolubility, hence resulting in a positive tone resist.

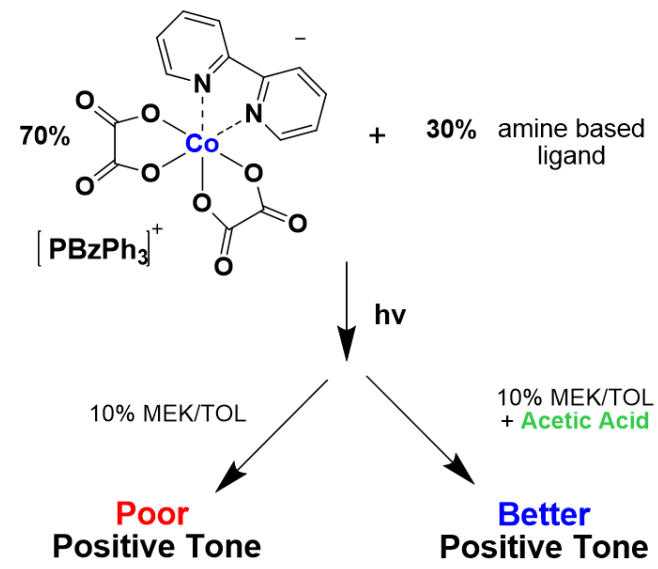

Theory:

The acid was able to break the oxalate bridging in, and increase the solubility of, the exposed regions.

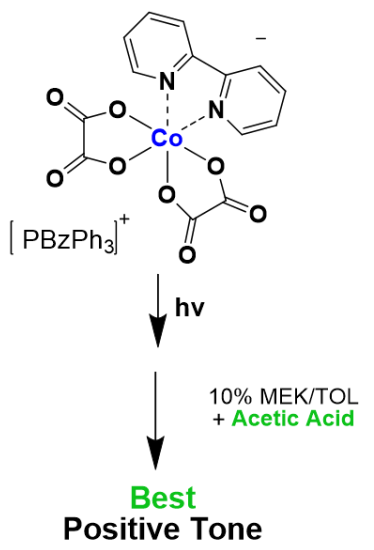

Figure 4. Schematic of the initial discovery of positive-tone behavior with NP1 (2).

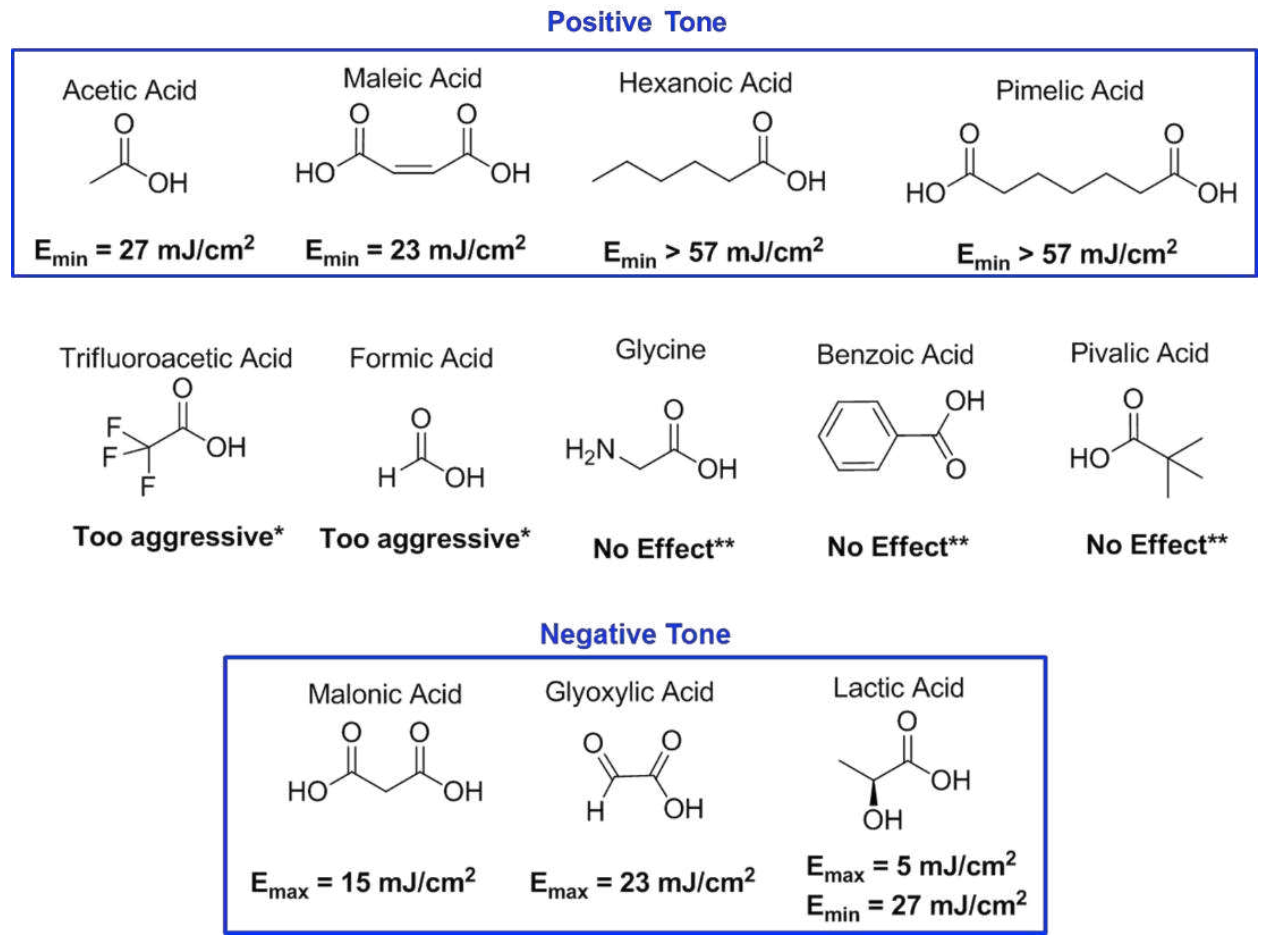

Figure 5. Structures of various organic acids. *These acidic developers dissolved both the exposed and unexposed regions at equal rates and almost immediately. ${ }^{*}$ These acidic developers do not dissolve either region within the development time. 
Only a few carboxylic acid developers afforded interesting results with NP1 (2): acetic, hexanoic, lactic, maleic and pimelic (Figure 6). The acidic developers studied were unable to fully clear the exposed regions of this resist. This phenomenon is independent of acid concentration and development time; there is approximately $30-40 \%$ of the film remaining after development. At the end of this section, we will speculate as to what is causing this residual thickness in the exposed regions.
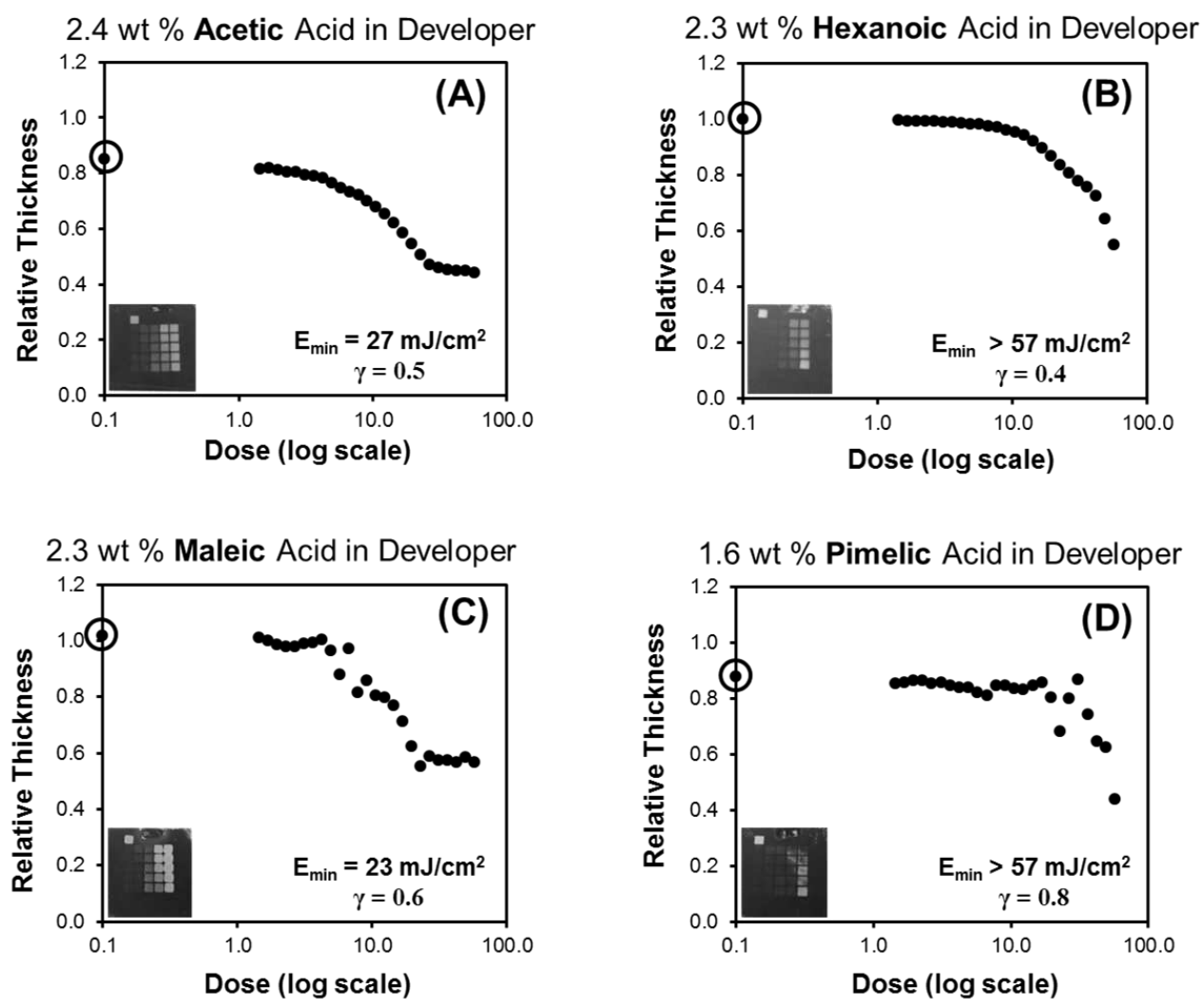

Figure 6. Contrast curve graphs of NP1 (2) with four different carboxylic acid developers. ${ }^{16}$

The term $\mathrm{E}_{0}$ is used to describe the dose required for a positive tone resist to fully clear, or zero thickness remaining, in the exposed region. However, these positive tone results all have scum remaining in the exposed regions after development. Therefore, we use the term $\mathrm{E}_{\min }$ to represent the dose required to achieve minimum thickness, not zero thickness.

The development of NP1 with acetic acid developer has a large process window. Variation in development time and concentration does not have a large impact on the thickness remaining in the exposed regions nor the contrast (Figure 7). Furthermore, when developed in acetic acid developer, this resist exhibits a large dose latitude: a small change in feature size when the dose is more than doubled (Figure 8). 
Developer: 2.4 wt \% Acetic Acid in Solution

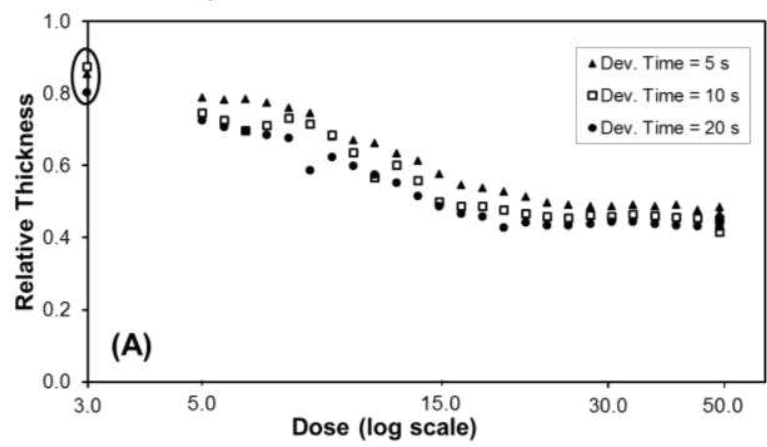

Development Time: $20 \mathrm{~s}$

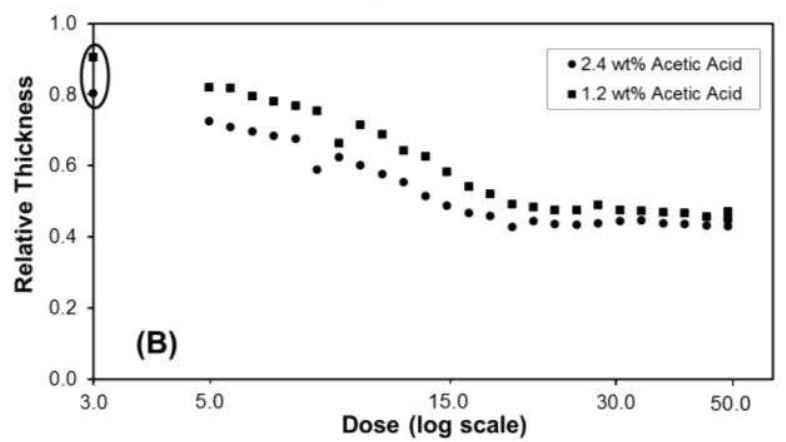

Figure 7. Variation in development time (graph A) or concentration of acid (graph B) does not significantly impact the contrast or the thickness remaining. ${ }^{16}$

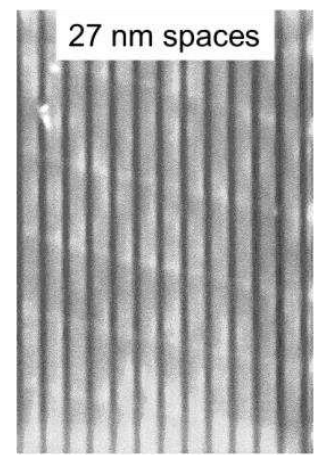

Dose:
$54 \mathrm{~mJ} / \mathrm{cm}^{2}$

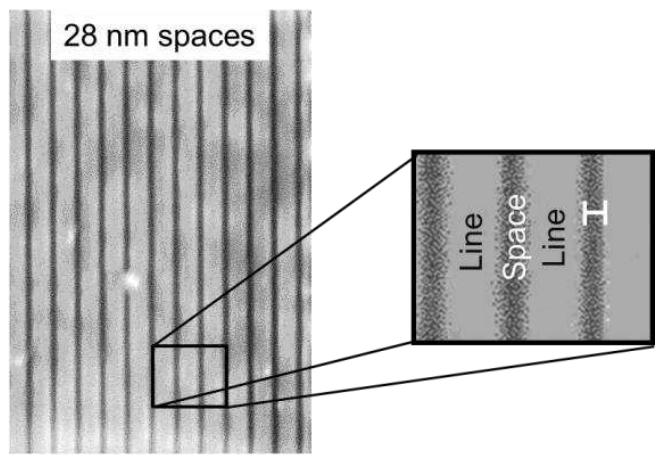

$118 \mathrm{~mJ} / \mathrm{cm}^{2}$

Figure 8. These patterned lines and spaces with NP1 compare the effect of dose on CD (critical dimension). Large variation in dose has a small effect on the CD size. These were printed using a mask with a $100 \mathrm{~nm}$ pitch with equal lines and spaces. Development: $1.2 \mathrm{wt} \%$ acetic acid in $10 \mathrm{wt} \%$ 2-butanone (MEK) and toluene for $30 \mathrm{~s}$.

We propose that the acid in the developer is acting as a ligand and displacing the oxalate bridging between cobalt centers. The benzyltriphenylphosphonium oxalate salt, $(\mathrm{BTP})_{2}(\mathrm{ox})$, could be the remaining thickness in the exposed regions (Figure 9). Additionally, as the concentration of certain acids in developer increases, we have seen switching from positive- to negative-tone imaging. We propose that at higher concentrations, a second molecule of acid reacts with the cobalt center to produce highly-ionic salts which we propose would be even less soluble in acidic non-polar solvents than the starting material (NP1, 2, Figure 9). 


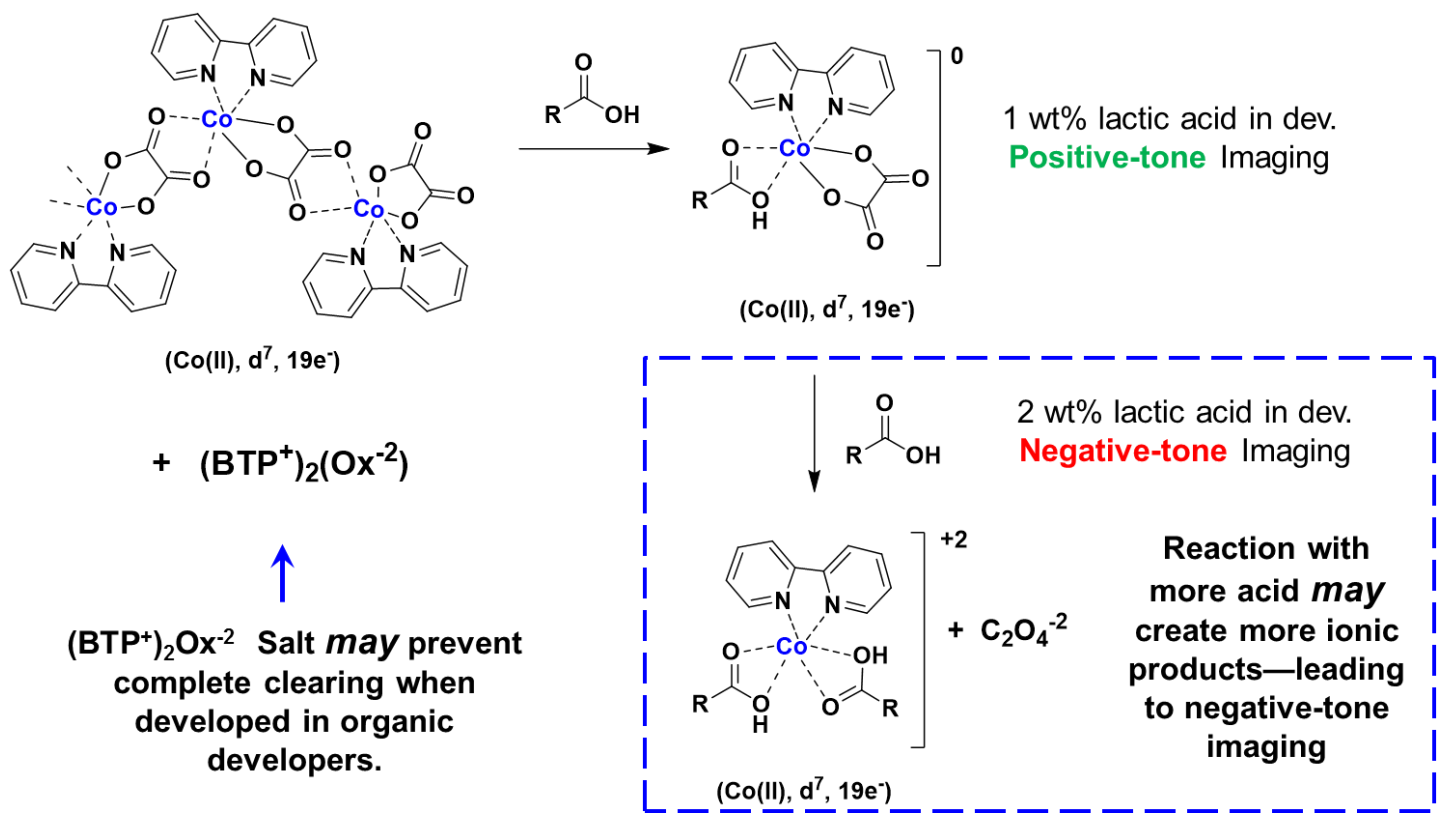

Figure 9. Hypothetical developer mechanisms.

\subsection{Organic Development: Negative-Tone Imaging of Cobalt Complexes}

The cobalt-oxalate complex, NP1, results in negative-tone imaging when developed in 2-butanone. In an attempt to optimize imaging capabilities of this resist we found that both contrast and photo-speed are improved simultaneously when cyclohexanone is used as the developer (Figure 10). The photoresist contrast was improved from 0.4 to 1.3 , and the sensitivity from 36 to $27 \mathrm{~mJ} / \mathrm{cm}^{2}$. In addition, the amount of top-loss, unintentional and unwanted film loss in the exposed regions of negative-tone imaging, was reduced from $35 \%$ down to $5-10 \%$.

\begin{tabular}{|c|c|c|}
\hline $\begin{array}{c}\text { Exposure } \\
\text { Chip: }\end{array}$ & \multicolumn{2}{|c|}{$\mathrm{NP} 1$} \\
\hline Resist: & \multicolumn{2}{|c|}{} \\
\hline $\begin{array}{c}\mathrm{E}_{\mathrm{max}} \\
\left(\mathrm{mJ} / \mathrm{cm}^{2}\right):\end{array}$ & $\mathbf{3 6}$ & $\mathbf{2 7}$ \\
\hline $\begin{array}{c}\text { Development: } \\
\text { Development } \\
\text { Time: }\end{array}$ & $\mathbf{2 - b u t a n o n e}$ & Cyclohexanone \\
\hline $\begin{array}{c}\text { Photoresist } \\
\text { Contrast }(\mathrm{y}):\end{array}$ & $\mathbf{0 . 4}$ & $\mathbf{3 0 ~ s}$ \\
\hline
\end{tabular}

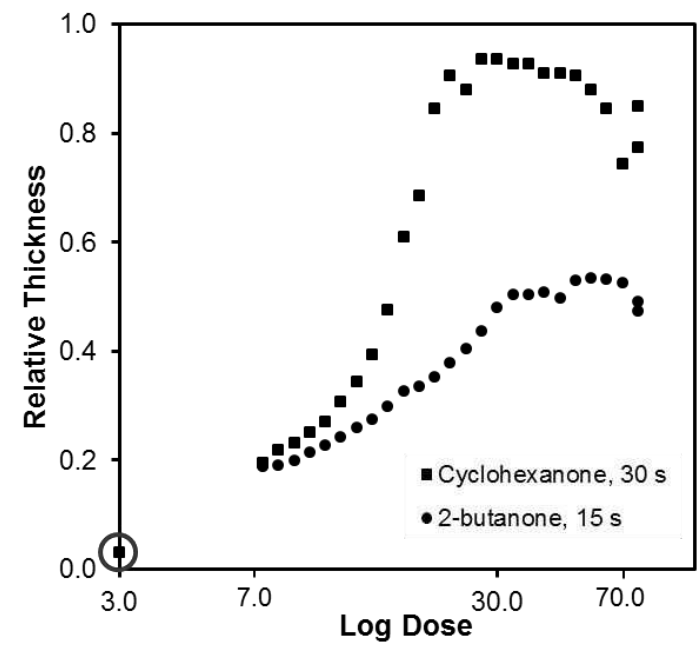

Figure 10. Best negative-tone sensitivity and contrast achieved with pure organic solvents as development of NP1 in pure organic solvents. ${ }^{16}$ 


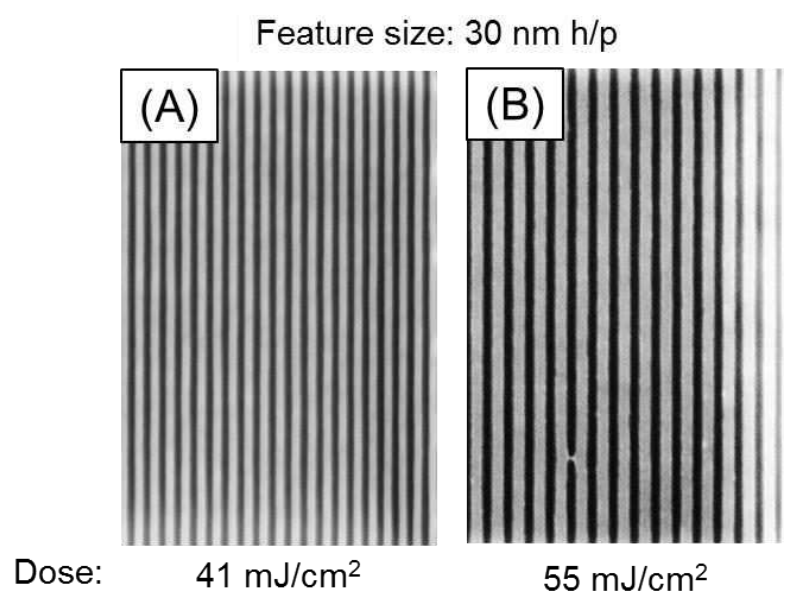

Figure 11. Patterned lines and spaces with NP1 comparing development. Development of A was with 2-butanone (15 s) and B was with cyclohexanone (45 s).

\subsection{Acidic Development: Negative-Tone Imaging of Cobalt Complexes}

With certain acidic developers, varying the concentration of the carboxylic acid affected the tone of the resist; lactic acid is one example of this. As the concentration of the lactic acid solution increases, the resist switches from positive- to negative-tone imaging (Graph A, Figure 12). The photoresist contrast of the negative-tone result of NP1 with development in a lactic acid solution is nearly 35 times higher than the original development in 2-butanone (MEK) (Graph B, Figure 12); we also see a large improvement in sensitivity from 36 to $5 \mathrm{~mJ} / \mathrm{cm}^{2}$. Last, when comparing this negative-tone result with lactic acid to an open source resist (OS2), though there is no difference in sensitivity, there is still a large improvement in contrast $(\gamma(\mathrm{OS} 2)=5.3, \gamma(\mathrm{NP} 1)=13.8)$.
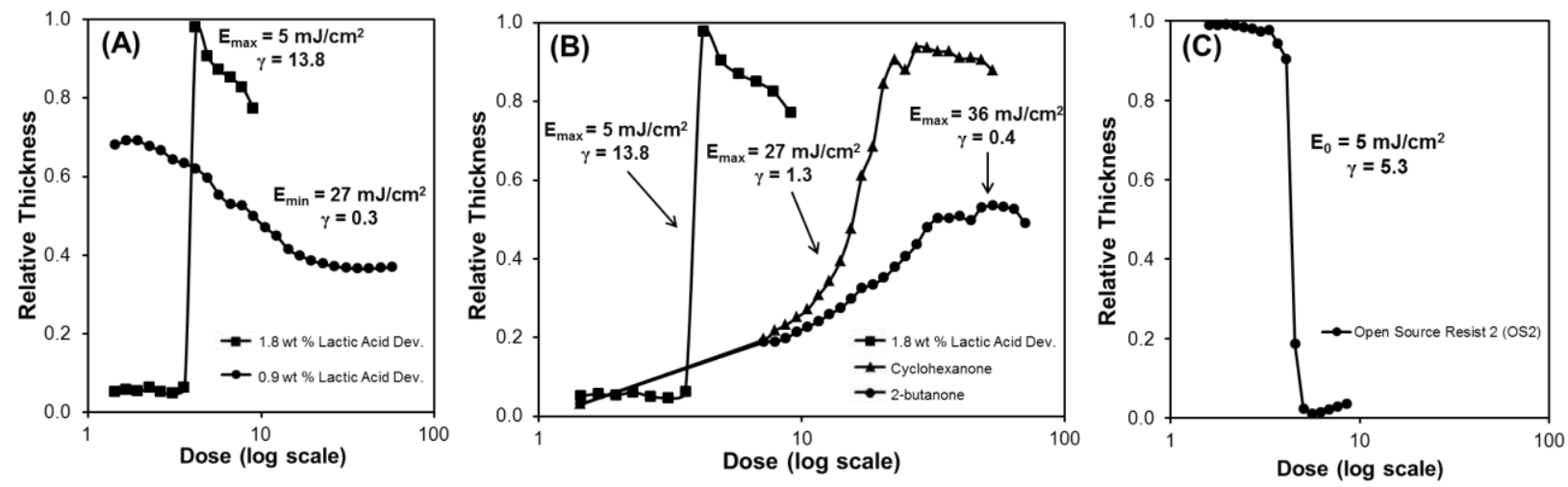

Figure 12. Contrast curves. Graph A compares development of NP1 with two concentrations $(0.9$ and 1.8 wt $\%)$ of lactic acid solutions. Graph B compares the development of NP1 in a $1.8 \mathrm{wt} \%$ lactic acid solution (5 s) to 2-butanone (MEK) (15 s) and cyclohexanone (30 s). Graph $\mathrm{C}$ is the contrast curve an open source resist (OS2) developed in tetramethylammonium hydroxide (TMAH) (45 s) and rinsed with water.

\subsection{Acidic Development: High-Speed MORE Systems}

We found that acidic solutions provided excellent development conditions for certain resists that would not develop in pure organic solvents. In the past, we tried developing these resists with many organic solvents with no success; a few are shown below (Figure 13). These resists are easily coated from organic solvents, and the freshly coated films can be redissolved in organic solvents, although after we expose these chips, the unexposed areas are no longer soluble. We suspected either flare or vacuum effects caused these changes in solubility. We ran a vacuum 
experiment in which one chip of each resist was kept at room pressure and temperature and another chip was placed under vacuum but not exposed. The chips were kept at each condition for equal lengths of time. All the chips were then developed at the same time in the same developer. We found that films that were subjected to vacuum pressures of approximately $10^{-6}$ to $10^{-7}$ torr, were no longer soluble in the same solvents even with extended development times. However, we found that vacuum-treated resist films could be successfully developed with solutions of carboxylic acids. Most of these resists exhibit fast photo-speeds with the carboxylic acid developers (Figure 14). We theorize that the sensitivities could be controlled by the concentration of acid in the developer. The graphs exhibit a two-part contrast curve (Figure 14). This phenomenon is not fully understood and is currently being investigated.

\section{Resist A}

\section{Chip 1}

Chip 2

Chip 3

Chip 4

\begin{tabular}{|c|c|c|c|c|}
\hline $\begin{array}{c}\text { Exposure } \\
\text { Chip: }\end{array}$ & & & & \\
\hline Development: & MEK, 60s & Toluene, 60s & $\begin{array}{c}2.3 \% \text { Hexanoic } \\
\text { Acid Dev., 45s }\end{array}$ & $\begin{array}{c}2.3 \% \text { Hexanoic } \\
\text { Acid Dev., 45s }\end{array}$ \\
\hline $\begin{array}{c}\text { Developer } \\
\text { Solvents: }\end{array}$ & -- & -- & $\begin{array}{c}10 \% \text { MEK in } \\
\text { Toluene }\end{array}$ & Hexanes \\
\hline Rinse: & -- & -- & Toluene & Hexanes \\
\hline
\end{tabular}

Chip 3

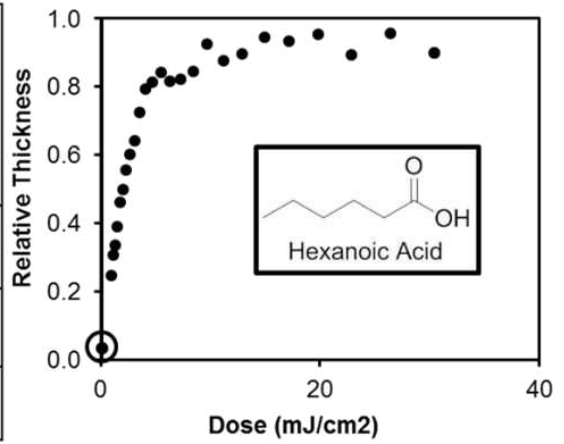

Figure 13. Solubility difference of resist A with organic developers and acidic developers. ${ }^{16}$
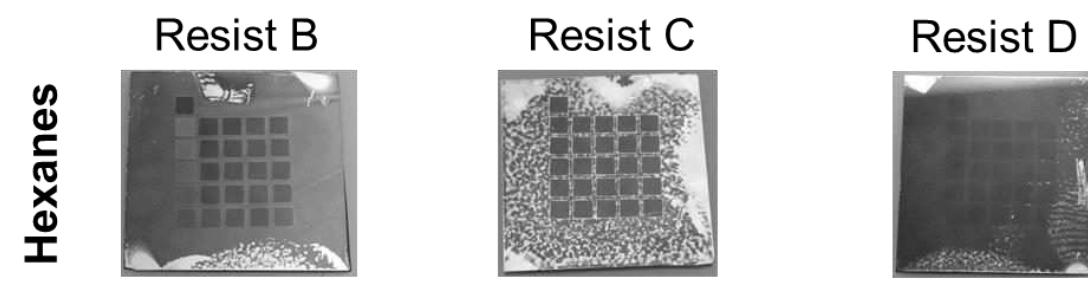

Resist E
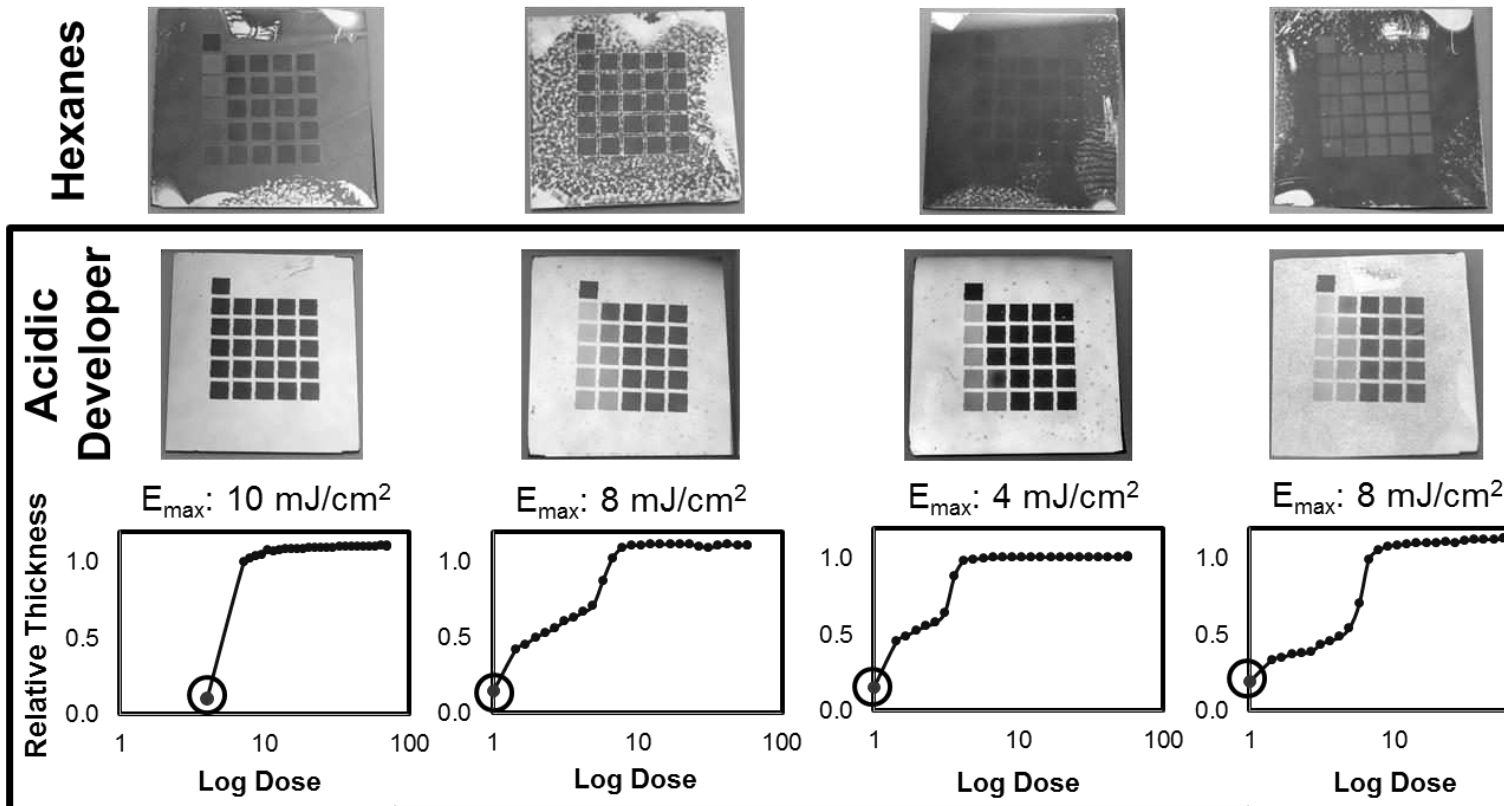

$E_{\max }: 8 \mathrm{~mJ} / \mathrm{cm}^{2}$
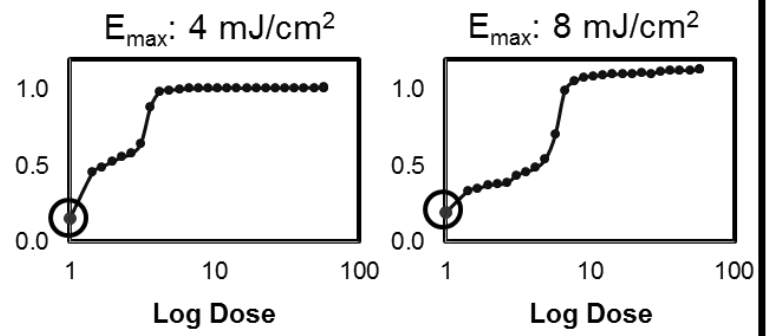

$2 \mathrm{~mL}$ Acid Developer in $18 \mathrm{~mL}$ Hex. with Hex. rinse

Figure 14. Contrast curves of other MORE resists (B-E) that were insoluble in organic solvents. First lithographic evaluation was using acidic developers. ${ }^{16}$ The development times for resists B-E were 60, 20, 20, and 60 seconds for hexanes, respectively, and 5, 5, 20, and 5 seconds for the acidic developer, respectively. 


\section{CONCLUSION}

In the past, we focused on designing new resists to enhance the photochemistry, not on understanding the chemical reactions occurring during development. We successfully achieved positive-tone imaging with the cobaltoxalate resist by using acidic developers. We suspect that the carboxylic acid in the developer is displacing the oxalate bridging between the cobalt centers. One challenge with the positive-tone imaging of NP1 is the inability to fully clear the exposed regions. We suspect that benzyltriphenylphosphonium oxalate salt, $(\mathrm{BTP})_{2}(\mathrm{ox})$, could be the remaining thickness in the exposed regions.

Development in cyclohexanone improved both the sensitivity from $E_{\max }=36$ to $27 \mathrm{~mJ} / \mathrm{cm}^{2}$ and the contrast from $\lambda=0.4$ to 1.3 of the negative-tone imaging of NP1. Some carboxylic acid developers, such as lactic acid, resulted in negative-tone imaging. The negative-tone contrast curves of NP1 with lactic acid development gave far better lithographic performance $\left(\mathrm{E}_{\max }=5 \mathrm{~mJ} / \mathrm{cm}^{2}\right.$ and $\left.\gamma=13.8\right)$ than that with pure organic solvents as developers. This photoresist contrast is 3 times greater than that of OS2, CAR resist used as a control, which was around $\gamma=5.3$.

Lastly, we successfully developed antimony-based resists A-E with carboxylic acid solutions. These resists were insoluble in common organic solvents after being under vacuum, and therefore never evaluated lithographically until now.

\section{EXPERIMENTAL METHODS}

4.1 General. Benzyltriphenylphosphonium bipyridinebis(oxalate)cobaltate(III) was synthesized following an experimental procedure found in literature that we modified. ${ }^{8}$ All reagents were either purchase through Alfa Aesar or Sigma Aldrich. The reagents were used as received unless otherwise specified.

\subsection{Resist formulation and imaging}

NP1 was solubilized in 1:2 acetonitrile to ethyl lactate. Resists A-E were spin-cast from a halogenated solvent. The formulations were filtered through a $0.2 \mu \mathrm{m}$ PTFE (polytetrafluoroethylene) filter. Formulations were spin-coated onto a custom underlayer (crosslinked hydroxyethyl methacrylate/methyl methacrylate copolymers) on 4-inch silicon wafers. Underlayer film thicknesses were around $20 \mathrm{~nm}$, and resist films varied from 40 to $60 \mathrm{~nm}$ by adjusting the spin-speed and formulation concentration. Resist films were then exposed with $13.5 \mathrm{~nm}$ radiation and developed with a carboxylic acid solution or pure organic solvent. Exposures were performed at the Paul Scherrer Institut (PSI) on the Swiss Light Source (SLS) XIL-II beamline using interference lithography, as well as the Lawrence Berkeley National Labs (LBNL) on the Berkeley Microfield Exposure Tool (BMET).

4.3 Developer formulation. Developers were formulated with 0.5-5 wt $\%$ acid, $10 \mathrm{wt} \%$ 2-butanone, and 85-89.5 wt $\%$ toluene. Certain carboxylic acids (e.g. maleic, glyoxylic, pimelic, and malonic) required diethyl ether or ethanol in place of 2-butanone to improve solubility.

\subsection{Metrology: ellipsometry, contrast, and SEM}

Ellipsometry. Thickness data was measured using a J. A. Woollam M-2000 ellipsometer. This ellipsometer has a fixed angle base that is at an incident angle of 65 degrees. Data was fitted with a cauchy model and analyzed using CompleteEase software.

Contrast curve analysis. The relative thickness after exposure and development was plotted as a function of the exposure dose on a logarithmic scale. The slope of the contrast region, refered to as photoresist contrast $(\gamma)$, 
was calculated by dividing the difference in relative thicknesses (t) by the difference in logarithmic (base-10) exposure doses (D) from the onset dose to $\mathrm{E}_{\max }$ or $\mathrm{E}_{0}$, such that

$$
\gamma=\frac{t_{1}-t_{0}}{\left|\log _{10}\left(\frac{D_{1}}{D_{0}}\right)\right|}
$$

The onset dose is the dose at which the resist starts to exhibit a solubility change. ${ }^{17,18}$

SEM metrology. Scanning electron microscope micrographs were collected at the Paul Scherrer Institut on a Zeiss Supra VP55 and at SUNY Polytechnic Institute on a Zeiss 1550. Accelerating voltage was set between 1-2 KeV and the working distance was 4-5 mm. All images were taken near $200 \mathrm{kx}$ magnification.

\section{ACKNOWLEDGMENTS}

We would like to thank Andrew Grenville and Stephen Meyers at Inpria for the financial support and scientific guidance of this work. We would also like to thank Dupont for supplying us with ESCAP polymer and King Industries for supplying us with a thermal acid generator for organic underlayers. In addition, we would like to thank Dr. Gregory Denbeaux and his graduate student, Steven Grzeskowiak, for the use of the J. A. Woollam M-2000 ellipsometer. Lithographic exposures were performed at the Paul Scherrer Institut (PSI) using the Swiss Light Source (SLS) and at the Lawrence Berkeley National Laboratory (LBNL) on the Berkeley Microfield Exposure Tool (BMET). We would like to thank Michaela Vockenhuber from PSI and Chris Anderson from LBNL for their assistance with EUV experiments. Finally, we would like to thank Patrick Naulleau at the Lawrence Berkeley National Laboratory for providing us with SuMMIT Lithography Image Analysis software.

\section{REFERENCES}

[1] Turkot, B., Carson, S.L., Lio, A., Liang, T., Phillips, M., McCool, B., Stenehjem, E., Crimmins, T., Zhang, G. and Sivakumar, S., "EUV progress toward HVM readiness," Proc. SPIE 9776, 977602 (2016).

[2] Mack, C. A., Thackeray, J. W., Biafore, J. J., and Smith, M. D., "Stochastic exposure kinetics of extreme ultraviolet photoresists: simulation study" J. Micro. Nanolithogr. MEMS MOEMS 10(3), 033019 (2011).

[3] Fallica, R., Stowers, J. K., Grenville, A., Frommhold, A., Robinson, A. P., \& Ekinci, Y., "Dynamic absorption coefficients of CAR and non-CAR resists at EUV" Proc. SPIE 9776, 977612 (2016).

[4] Brainard, R. L., "Photoresists for extreme ultraviolet lithography", EUV lithography. SPIE Press, (2009).

[5] Stowers, J. and Keszler, D. A., "High resolution, high sensitivity inorganic resists." Microelectron. Eng. 86(4), 730-733 (2009).

[6] Ober, C. and Giannelis, E., "New oxide nanoparticle extreme-UV photoresists achieve high sensitivity" SPIE Newsroom, (2014).

[7] Grenville, Andrew, et al. "Integrated fab process for metal oxide EUV photoresist" Proc. SPIE 9425, 94250S (2015).

[8] Miles Marnell, Brian Cardineau, Hashim Al-Mashat, Kara Heard, Amber Aslam, Jason Pavlich, Ryan Del Re, Michael Vockenhuber, Yasin Ekinci, Chandra Sarma, Mark Neissar, Robert Brainard and Dan Freedman, "A molecular inorganic approach to EUV photoresists", presented at SPIE advanced lithography conference, February 2014, San Jose CA.

[9] Del Re, R., Passarelli, J., Sortland, M., Cardineau, B., Ekinci, Y., Buitrago, E., Neisser, M., Freedman, D. A. and Brainard, R. L., "Low-line edge roughness extreme ultraviolet photoresists of organotin carboxylates", J. Micro. Nanolithogr. MEMS MOEMS 14(4), 043506 (2015).

[10] Sortland, M., Del Re, R., Passarelli, J., Hotalen, J., Vockenhuber, M., Ekinci, Y., Neisser, M., Freedman, D. A. and Brainard, R. L., "Positive-tone EUV resists: complexes of platinum and palladium." Proc. SPIE 94222, 942227(2015). 
[11] Passarelli, J., Sortland, M., Del Re, R., Cardineau, B., Sarma, C., Freedman, D. A. and Brainard, R. L., "Bismuth resists for EUV lithography" J. Photopolym. Sci.Technol. 27(5), 655-661 (2014).

[12] Passarelli, J., Murphy, M., Del Re, R., Sortland, M., Hotalen, J., Dousharm, L., Fallica, R., Ekinci, Y., Neisser, M., Freedman, D. A. and Brainard, R. L., "Organometallic carboxylate resists for extreme ultraviolet with high sensitivity" J. Micro. Nanolithogr. MEMS MOEMS 14(4), 043503-043503, (2015)

[13] Grzeskowiak, S., Narasimhan, A., Murphy, M., Ackerman, C., Kaminsky, J., Brainard, R. L., Denbeaux, G., "Reactivity of metal oxalate EUV resists as a function of the central metal" presented at SPIE advanced lithography conference, (Feb. 2017). (Publication Pending)

[14] Narasimhan, A., Grzeskowiak, S., Srivats, B., Herbol, H., Wisehart, L., Schad, J., Kelly, C., Earley, W., Ocola, L. E., Neisser, M. and Denbeaux, G., "Studying thickness loss in extreme ultraviolet resists due to electron beam exposure using experiment and modeling" J. Micro. Nanolithogr. MEMS MOEMS 14(4), 043502-043502 (2015).

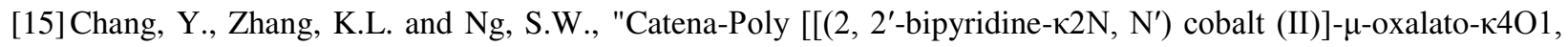
O2: O1', O2']" Acta Crystallographica Section E: Structure Reports Online 65(10), m1243 (2009).

[16] Circled data points are the thicknesses at dose equal to zero (unexposed thickness) but placed arbitrarily at a dose above 1 to be shown on the log scale.

[17] Mack, C., Fundamental Principles of Optical Lithography: The Science of Microfabrication. John Wiley \& Sons, 2008.

[18] Kirchauer, H., 2.6.1 Contrast and Important Properties. Institute for Microelectronics TU Vienna, (1998), http://www.iue.tuwien.ac.at/phd/kirchauer/node29.html. Accessed 23 Aug. 2016. 